\title{
Oscillator Strengths for Neutral, Charged, and Scattered Excitons in CdTe Quantum Wells Containing a 2D Electron Gas
}

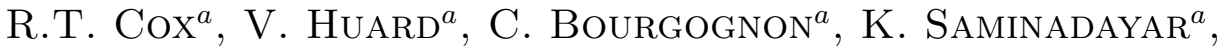 \\ S. TATAREnKo ${ }^{a}$ AND R.B. Miller ${ }^{b}$ \\ ${ }^{a}$ Equipe "Nanophysique et Semiconducteurs" de SP2M, CEA-Grenoble \\ et du Laboratoire de Spectrométrie Physique, Université Joseph Fourier \\ 38054 Grenoble-Cedex, France \\ ${ }^{b}$ Department of Physics, La Trobe University, Victoria 3086, Australia
}

\begin{abstract}
Absorption spectra have been measured for a $10 \mathrm{~nm} \operatorname{CdTe}(0.3 \% \mathrm{Mn})$ quantum well with electron concentration $n_{\mathrm{e}}$ variable up to $1.5 \times 10^{11} \mathrm{~cm}^{-2}$. Following recent theory appropriate to low $n_{\mathrm{e}}$, here $\approx 0.1 / \pi a_{\mathrm{B}}^{2}$, the spectra are interpreted in a "strong exciton" model, where the initial oscillator strength of the excitonic resonance $(X)$ is conserved, with screening and phase-space filling effects negligible. As $n_{\mathrm{e}}$ increases in zero-field and as the filling factor $\nu$ increases in magnetic field, the intensity of $X$ is transferred to: (i) trion processes, namely exciton-one electron scattering and the trion resonance $(T)$, and (ii) quatron processes, namely exciton-two electron scattering and trion-one electron scattering. In magnetic field, the three- and four-body scattering processes become discrete, combined "exciton and cyclotron" and combined "trion and cyclotron" excitations that take all the intensity of $X$ and $T$ for $\nu \gtrsim 1$ and $\nu>2$, respectively.
\end{abstract}

PACS numbers: 78.30.Fs, 78.67.De, 78.40.Fy, 78.55.Et

\section{Introduction}

The linear absorption spectra of modulation doped quantum wells (QWs) near the band-gap energy are reasonably well understood for very low electron or hole concentrations, $n_{\mathrm{e}}$ or $n_{\mathrm{h}} \ll 1 / \pi a_{\mathrm{B}}^{2}$, where $a_{\mathrm{B}}$ is the radius of the quasi 
2D exciton. Much less well understood are the absorption properties at somewhat higher concentrations that we will call "low", as distinct from very low (and without attempting here to define any upper limit). By "low" we mean carrier concentrations $n$ around $1 \times 10^{11} \mathrm{~cm}^{-2}$ for CdTe QWs with quasi-2D $a_{\mathrm{B}} \approx 5 \mathrm{~nm}$, that is $n \approx 0.1 / \pi a_{\mathrm{B}}^{2}$ (equivalent to $n \approx 3 \times 10^{10} \mathrm{~cm}^{-2}$ in GaAs QWs).

This paper is an attempt to develop a new understanding of the absorption spectra seen in this low concentration range. We will first recall the basic absorption properties at very low carrier concentration (for more information see the review [1]), simplifying to mention only heavy-hole transitions, thus:

In zero-magnetic field, the absorption spectrum is dominated initially by a single resonance peak $(X)$ corresponding to the creation of $1 s$ excitons. As carriers are added to the well, a second resonance $(T)$ appears below $X$, corresponding to the creation of spin singlet trions $\left(X^{-}\right.$in $n$-type samples, $X^{+}$in $p$-type), while the peak $X$ broadens asymetrically to the high energy side due to exciton-carrier scattering. The peaks $X$ and $T$ are separated in energy by the trion binding energy $E_{\mathrm{b} 1}$.

In a magnetic field $B$, the energy of the peak $X$ varies quadratically with $B$ at low $B$. It can end up following the linear variation of the lowest Landau bandgap if the combined cyclotron energy $\hbar \omega_{\text {ce }}+\hbar \omega_{\text {ch }}$ becomes $\gg E_{\mathrm{b} 2}$ (the exciton binding energy). The (singlet) trion peak $T$ may show a red-shift initially, and then follows the $X$ peak quite closely. A triplet trion peak can resolve out between $X$ and $T$. The scattering wing of $X$ resolves (in $n$-type QWs at least) into the "combined exciton and cyclotron resonance" $\left(X \& C R_{i}\right)$ excitations discovered by Yakovlev et al. [2]: these peaks lie above the peak $X$ at separation $i \hbar \omega_{\text {ce }}(i=1,2, \ldots)$, multiplied by a momentum-conserving, exciton-recoil factor of about $1+m_{\mathrm{e}} / M_{X}$.

The above-mentioned properties have been elucidated in recent theoretical work [3-6] on the optical properties of a 2D semiconductor with carrier concentration low enough that the probability of presence of a background carrier within the radius of the exciton remains $\ll 1$. These recent theoretical methods, retaining only processes linear in carrier concentration, emphasize essential correlations of three bodies (namely the photo-electron, the photo-hole, and just one background carrier) that are not captured by the mean-field, many-body methods $[7,8]$ appropriate to high carrier concentration $\gg 1 / \pi a_{\mathrm{B}}^{2}$.

In particular, Esser et al. have predicted that the three-body processes trion resonance and exciton-electron scattering - compete with the exciton resonance, sharing its oscillator strength with increasing carrier concentration [6]. We have confirmed this recently [9] by analysing spectra of electron doped CdTe QWs with $n_{\mathrm{e}}$ up to $1 \times 10^{11} \mathrm{~cm}^{-2}$ : integrated over the wavelength band corresponding to $X, T$, and exciton-electron scattering, less than $10 \%$ of the absorption intensity was lost as $n_{\mathrm{e}}$ increased up to $1 \times 10^{11} \mathrm{~cm}^{-2}$, at both zero field and $B=8 \mathrm{~T}$.

In this article, we discuss important spectroscopic features additional to those mentioned above, that become very evident when the carrier concentration 
is increased from very low into the "low" carrier concentration range. We will argue that these additional properties can still be interpreted in terms of few-body excitations involving a strongly bound exciton.

The first additional effect is absorption features associated with four-body interactions, called "quatron" processes by Esser who outlined a four-body theory in Ref. [10]. These processes are (i) trion creation with scattering of a second carrier becoming, under applied magnetic field, the "combined trion and cyclotron resonance" ( $T \& C R)$ excitations identified by Kochereshko et al. [11], and (ii) exciton creation with scattering of two carriers. The second additional effect is anomalies in the energies of the absorption peaks showing that the few-body processes are nevertheless "dressed" by a background Fermi sea: carrier capture into the trion must leave a hole in the Fermi sea (as pointed out by Suris [12]), and scattering of a carrier must leave a hole in the Fermi sea and must take the carrier to unoccupied states that are all outside the Fermi sea (at low $T$ ).

Our measurements concern a QW sample M1120, called S6 here. It is a $10 \mathrm{~nm} \mathrm{Cd}_{0.997} \mathrm{Mn}_{0.003} \mathrm{Te}$ single quantum well between CdMgTe barriers, grown in our laboratory by molecular beam epitaxy on a $\mathrm{CdZnTe}(12 \% \mathrm{Zn})$ substrate. The substrate is transparent to $1640 \mathrm{meV}$, so that the absorption spectrum can be measured in the QW excitonic region around 1610-1620 meV. The substrate also imposes a large biaxial strain on the $\mathrm{CdTe}(0.3 \% \mathrm{Mn})$ well layer, splitting off light-hole transitions to high energies, simplifying the optical spectra.

The upper barrier is doped with a plane of aluminium donors at $60 \mathrm{~nm}$ from the well edge, giving $n_{\mathrm{e}}=1.5 \times 10^{11} \mathrm{~cm}^{-2}$ in the well in equilibrium. Due to specific properties of aluminium doping (not yet understood), $n_{\mathrm{e}}$ can be reduced by a factor of about three or four during the transmission measurements by pumping the sample with blue laser light. (Luminescence induced by the pump light has to be subtracted, by measuring with and without the tungsten lamp light that probes the transmission spectra.) The $0.3 \% \mathrm{Mn}$ added in the well gives large Zeeman splittings of the conduction band levels, which yields very well-defined spin polarization effects, helpful for understanding the magneto-absorption spectra.

\section{Absorption of a $\operatorname{CdTe}(0.3 \% \mathrm{Mn}) \mathrm{QW}$ in zero-field}

We begin by discussing zero-field spectra. Figure 1a shows $2 \mathrm{~K}$ absorption spectra in the excitonic region for sample $\mathrm{S} 6$, for various values of $n_{\mathrm{e}}$ obtained by varying the pumping light intensity. The scale of $n_{\mathrm{e}}$ is not yet calibrated properly but runs from approximately 0.4 to (accurately) $1.5 \times 10^{11} \mathrm{~cm}^{-2}$.

In Fig. 1a, the two absorption peaks, corresponding to trion $\left(X^{-}\right)$and exciton resonances at the lowest $n_{\mathrm{e}}$, are both strongly broadened at the highest $n_{\mathrm{e}}$. Also, their separation is increasing strongly with $n_{\mathrm{e}}$, becoming much greater than $E_{\mathrm{b} 1} \approx 2.3 \mathrm{meV}$. As in Refs. [13-15], the splitting is of the order of the Fermi energy $E_{\mathrm{F}}=n_{\mathrm{e}} \pi \hbar^{2} / m_{\mathrm{e}}=2.3 \mathrm{meV} / 10^{11} \mathrm{~cm}^{-2}$. Nearly all the shift is in the higher energy 


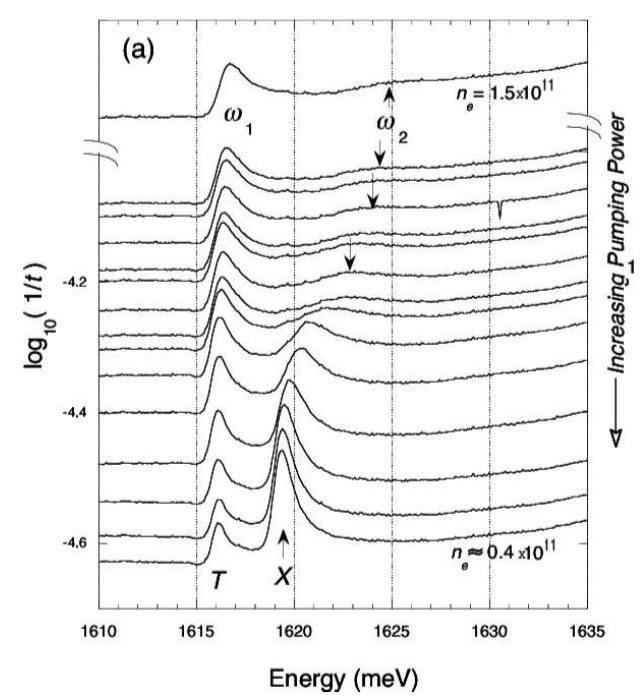

(b)

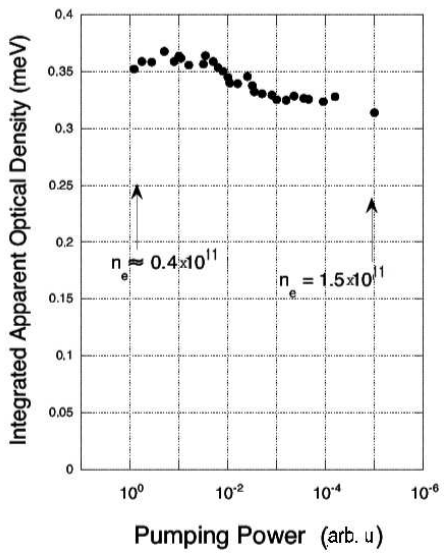

Fig. 1. (a) Optical absorption spectra $(\log (1 /$ transmission $))$ at $2 \mathrm{~K}$ in zero-field for $\operatorname{CdTe}(0.3 \% \mathrm{Mn}) / \mathrm{CdMgTe}$ QW sample S6. Values of $n_{\mathrm{e}}$ from $\approx 0.4 \times 10^{11}$ to $1.5 \times$ $10^{11} \mathrm{~cm}^{-2}$ (top spectrum, no pumping) are obtained by varying $488 \mathrm{~nm}$ pumping power. Spectra are shifted vertically by amounts $\propto \log (1 /$ pumping power $) ; n_{\mathrm{e}}$ variation is slow at bottom and top, rapid for spectra in middle. (b) Total absorption intensities integrated between 1613 and $1638 \mathrm{meV}$ in (a), after subtracting sloping background due to $\mathrm{CdZnTe}(12 \% \mathrm{Zn})$ substrate absorption.

peak; the lower peak shifts only slightly. We refer to the two peaks as $\omega_{1}$ (evolved from $T$ ) and $\omega_{2}$ (evolved from $X$ ), following Hawrylak's notation [8].

The data plotted in Fig. 1b show that, despite the great changes in width and shape of the two peaks as $n_{\mathrm{e}}$ increases, their summed integrated intensity hardly varies: the decrease in integrated intensity at $1.5 \times 10^{11} \mathrm{~cm}^{-2}$ does not exceed $15 \%$. This is provided we integrate right out to the end of the high energy wing of the $\omega_{2}$ peak (whose full extent at high $n_{\mathrm{e}}$ is revealed by subtracting the spectrum for minimum $n_{\mathrm{e}}$ to eliminate the low energy tail of the substrate's absorption).

At our highest $n_{\mathrm{e}}$, the $\omega_{2}$ peak has broadened very strongly and is almost lost in the background. In such cases the lower peak, $\omega_{1}$, has generally been interpreted as a "Fermi edge singularity" (FES). In the FES theory (see Ref. [7] and more recent work that includes electron spin $[8,16]$ ), the optical response peaks at the Fermi edge due to multiple small amplitude excitations involving very many background carriers near the Fermi level, as well as exchange with these carriers.

This has been our own interpretation of the $\omega_{1}$ peak previously [13]. But the FES theory was worked out for a high carrier concentration $\gtrsim 1 / \pi a_{\mathrm{B}}^{2}$, and we think now that a few-body explanation is more appropriate at low $n_{\mathrm{e}}$. Just as the trion resonance is a three-particle process, a broadened trion resonance, as seen 
in Fig. 1a, can be interpreted as the signature of four-body processes: a second electron can scatter off the trion, with recoil of the trion centre of mass.

Logically, the $\omega_{2}$ peak should then be considered as an exciton scattered by two electrons. The splitting between $\omega_{2}$ and $\omega_{1}$ peaks, which is $E_{\mathrm{b} 1}$ at vanishing $n_{\mathrm{e}}$, then contains the energy difference between the exciton - two-electron scattering and the trion - one-electron scattering.

As concerns the intensities, Fig. 1b implies a sum rule beyond Esser et al.'s calculation of summed intensities for three-particle processes [6]. This more general sum rule would include also four-particle processes, conserving quite accurately the excitonic oscillator strength within the excitonic region of the spectrum at both very low and "low" $n_{\mathrm{e}}$. At some high $n_{\mathrm{e}}$, the excitonic oscillator strength must eventually be quenched by phase-space filling and screening as per mean-field theory, but the transition to the latter situation awaits accurate experimental study.

\section{Magneto-absorption of a $\operatorname{CdTe}(0.3 \% \mathrm{Mn}) \mathrm{QW}$}

In our experience (with CdTe quantum wells), no matter how strong the carrier-induced broadening of the trion and exciton absorption in zero-field, sharp trion and exciton absorption peaks always re-emerge at sufficiently high magnetic field. The $T$ resonance appears first, at a filling factor thought to be precisely $\nu=2$ and the $X$ resonance appears later, around $\nu=1$. We will propose a new explanation of these effects that appears appropriate to the "low $n_{\mathrm{e}}$ " case.

\subsection{Spectra}

The emergence of $T$ and $X$ is seen clearly in Fig. 2 for sample $\mathrm{S} 6$ at its equilibrium $n_{\mathrm{e}}=1.5 \times 10^{11} \mathrm{~cm}^{-2}$ (i.e. no laser pumping). In both $\sigma^{-}$and $\sigma^{+}$ polarizations, the zero-field peak $\omega_{1}$ first breaks up into sharper peaks $G_{i}(i=$ $\ldots, 2,1)$ that move off towards high energy. Then, in $\sigma^{-}$(Fig. 2a), a sharp trion resonance $T$ emerges at $\nu=2(B=3 \mathrm{~T})$ and strengthens with increasing field. In $\sigma^{+}$(Fig. 2b) a sharp $X$ resonance emerges at $\nu=1(B=6 \mathrm{~T})$, and no $T$ resonance is seen. Also in $\sigma^{+}$, a peak labeled $Z_{1}$, appearing near $\nu=2$ and becoming a complex band at high $B$, corresponds to $X \& C R[2,9]$.

The $\sigma^{-}$polarization of the singlet trion creation transition, for $\nu<1$ and low temperature, comes from well-known selection rules [17], with the electron $g$-factor $g_{\mathrm{e}}$ positive for the case of CdMnTe. With the background electrons polarized spin-down here, spin singlet trions (i.e. with two anti-parallel electron spins) can be created only by $\sigma^{-}$photons, which inject spin-up photoelectrons.

But why is no $\sigma^{+}$trion resonance seen between $\nu=1$ and $\nu=2$, where there are background electrons in both spin states? Also, why does the $\sigma^{-}$trion resonance disappear (with decreasing $B$ ) at $\nu=2$ ? 


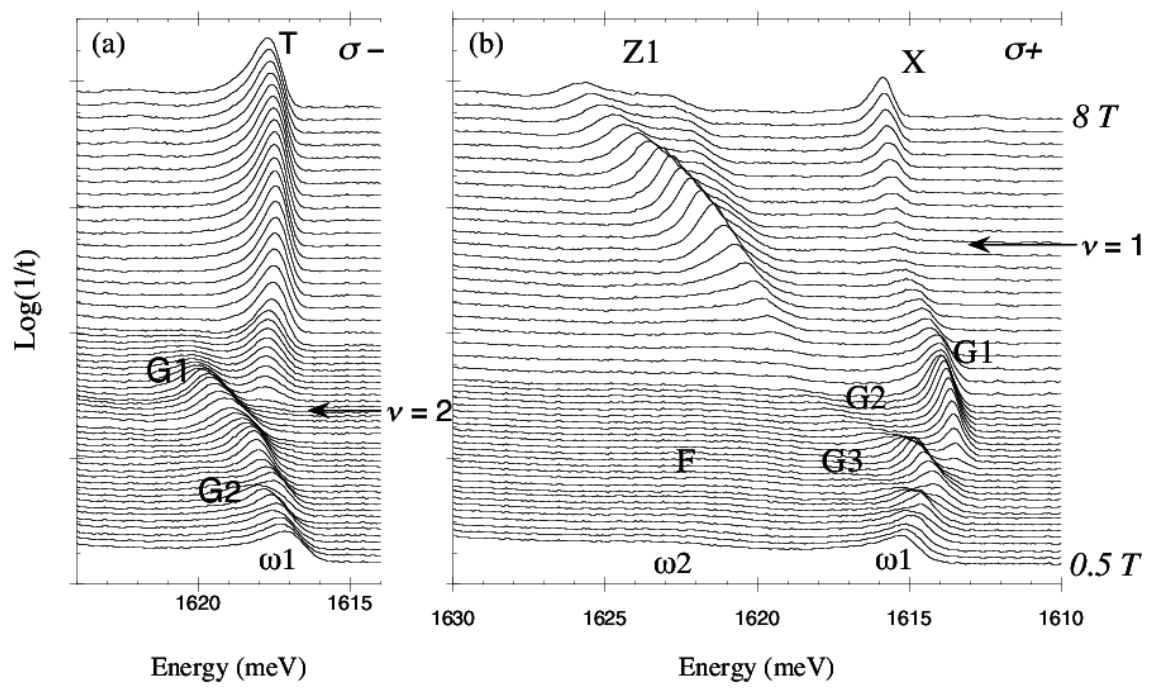

Fig. 2. Polarized optical absorption spectra $(\log (1 /$ transmission $))$ at $2 \mathrm{~K}$ for sample S6 with $n_{\mathrm{e}}=1.5 \times 10^{11} \mathrm{~cm}^{-2}$. Spectra are for magnetic fields from 0.5 to $8 \mathrm{~T}$. The $\sigma^{-}$ and $\sigma^{+}$branches are split by the giant Zeeman effect $(\approx 5 \mathrm{meV}$ at saturation). Peaks $X$ and $T$ are attributed to exciton and trion resonances, peaks $Z$ and $G$ to scatterings $X \& C R_{i}$ and $T \& C R_{i}$, respectively.

Until very recently, our answer to both these questions was "phase-space filling", see an (auto)critical discussion of this in Ref. [9]. But the data reported for CdTe QW samples S3 and S5 in [9] forced us to reattribute a similar progressive extinction of $X$, that occurs as $\nu$ increased up towards $\nu=1$, to intensity sharing with the $Z$ peaks $(=X \& C R)$. In an entirely analogous way, the present data leads us to attribute the extinction of $T$, as $\nu$ increases up to $\nu=2$, to intensity sharing with the $G$ peaks.

Let us first examine the peak labeled $G_{1}$ in Fig. 2 a and b. We identify $G_{1}$ with the combined trion and cyclotron resonance $(T \& C R)$ process of Kochereshko et al. [11]. This is a four-body or quatron process. The incident photon creates a trion that takes one background electron, and at the same time a second background electron is scattered from Landau level $n=0$ to Landau level $n=1$ (with trion recoil).

Our attribution is based on Fig. 3, which plots the energies of $\sigma^{-}$peaks $G_{1}$ and $T$ (with $T$ emerging at $\nu=2$ as noted earlier). As in Ref. [11] the slope of the $G_{1}-T$ splitting depends on how one fits the characteristic initial red-shift of $T$. We estimate a slope difference of $\approx 1.2 \mathrm{meV} / \mathrm{T}$, which is somewhat greater than $\hbar \omega_{\text {ce }}=1.11 \mathrm{meV} / \mathrm{T}$ and thus fits a $T \& C R$ process with some trion recoil.

But there is a striking anomaly in Fig. 3: the graphs for $G_{1}$ and $T$ (extrapolated) intersect around $B=1.5 \mathrm{~T}$ instead of at $0 \mathrm{~T}$, see later (Sec. 3.3). 


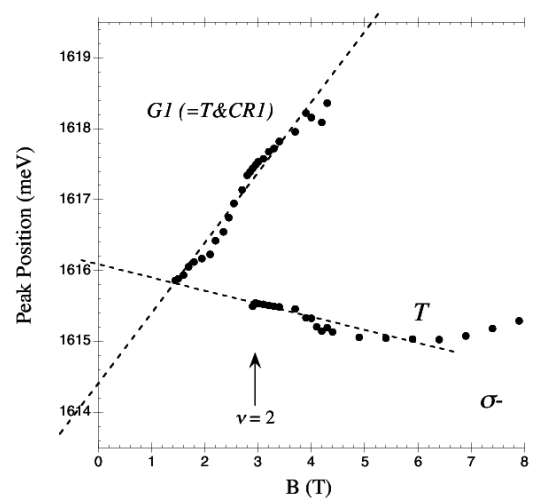

Fig. 3. Energy positions of peak $G_{1}$ (attributed to $T \& C R$ scattering) and $T$ (=trion resonance) seen in $\sigma^{-}$polarization, after subtracting Mn-related giant Zeeman shift. The difference between slopes of $G_{1}$ and $T$ is $\approx 1.2 \mathrm{meV} / \mathrm{T}$.
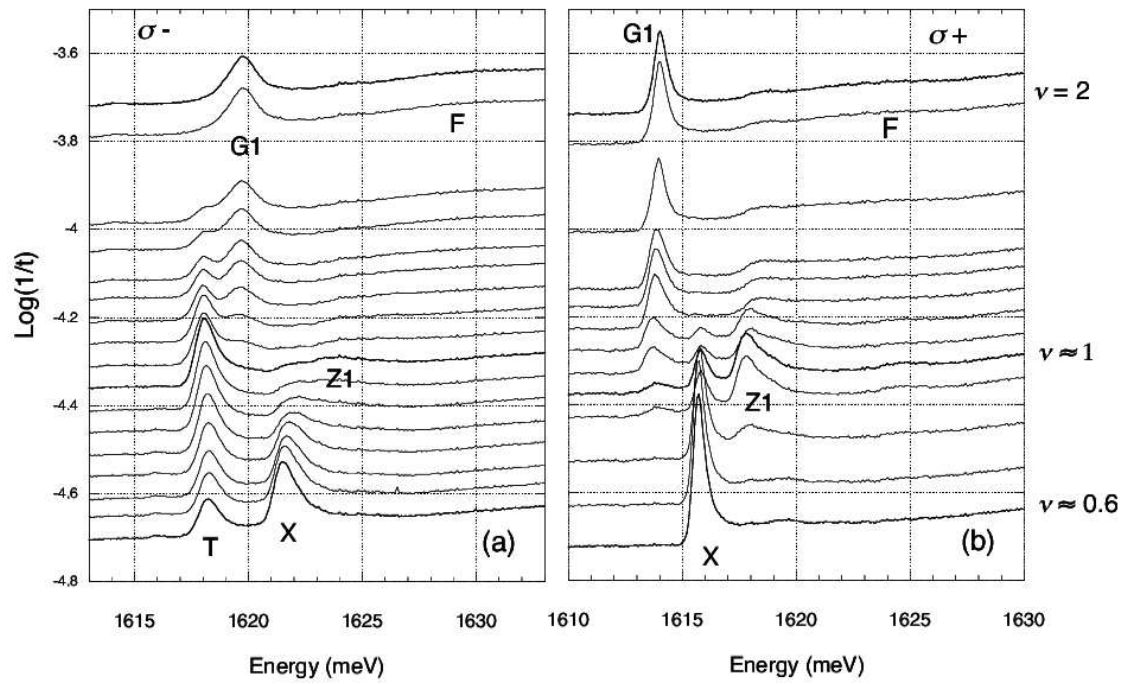

Fig. 4. Polarized optical absorption spectra ( $\log (1 /$ transmission $))$ for sample S6 in a fixed field of $2.9 \mathrm{~T}$. The range of $n_{\mathrm{e}}$ is the same as in Fig. 1; a filling factor varies from $\nu \approx 0.6$ at bottom up to $\nu=2$ at top. Spectra are shifted vertically by amounts $\propto \log (1 /$ pumping power $)$. Spectra at same level in (a) and (b) have the same $\nu$. Variation of $\nu$ is slow at bottom and top, fast in middle of the set of spectra.

Figure 4 gives a different view on the $\sigma^{-}$and $\sigma^{+}$magneto-absorption, namely the evolution as $n_{\mathrm{e}}$ varies at a fixed field $(B=2.9 \mathrm{~T})$. Here, $\nu$ ranges from $\approx 0.6$ up to $\nu=2$. Notice first that the energy positions of the different absorption peaks hardly change over this range. As concerns the intensities, the $\sigma^{-}$spectra (Fig. 4a) are the simplest to describe: (i) At small $\nu$ we have a trion resonance $T$ 
and a broadened $X$ resonance. (ii) As $n_{\mathrm{e}}$ increases, peak $X$ loses intensity, sharing it out mainly to peak $T$ and a little to $Z_{1}\left(=X \& C R_{1}\right)$. (iii) $T$ grows with $n_{\mathrm{e}}$, passes through a maximum near $\nu=1$ and decreases as $G_{1}\left(=T \& C R_{1}\right)$ appears. (iv) At $\nu=2$, peak $T$ disappears having transferred its intensity to $G_{1}$.

In $\sigma^{+}$(Fig. 4b), the $T$ resonance is never observed, at any $\nu$ (this would not be so for QWs with small $g_{\mathrm{e}}$, or at higher temperature). (i) At the lowest $n_{\mathrm{e}}$ we have a strong $X$ resonance, narrower than in $\sigma^{-}$. (ii) As $\nu$ increases, $X$ shares intensity out to $Z_{1}\left(=X \& C R_{1}\right)$. (iii) At higher $\nu$, peaks $X$ and $Z_{1}$ are sharing intensity out to peak $G_{1}\left(=T \& C R_{1}\right)$. Intensity also goes to peak $F$ at high energy, which we attribute to an $X \& 2 C R$ process (not to be confused with $X \& C R_{2}=$ scattering of one-electron from Landau level $n=0$ to $n=2$ ).

Integrating the absorption intensity over the spectrum of peaks $X, T, Z, G$, and $F$, we find that the total intensity decreases by less than $15 \%$ in either polarization over the available range of $n_{\mathrm{e}}$ up to $1.5 \times 10^{11} \mathrm{~cm}^{-2}$. That is, despite the complex changes in the number and nature of the observed transitions, the total oscillator strength in the excitonic region is quite accurately shared, just as in zero-field.

\subsection{Model}

Figure 5 summarizes our model for the magneto-absorption properties, up to $\nu=2$. We consider five processes, starting with the exciton resonance $X$ (left hand panel). Scatterings X\&CR and resonance $T$ are trion processes (central panel). Scatterings $X \& 2 C R$ and $T \& C R$ are quatron processes (right hand panel). To preserve charge neutrality, we must add holes to the $n=0$ Landau level for each electron displaced out of that level. Thus, four of the five processes are dressed by simultaneous creation of conduction band holes.

Why do the five processes appear/disappear with changes of the filling factor $\nu$ ? In answer to this, we take Esser's basic premise $[6,18]$ : "the generation of an electron-hole pair may occur either with a carrier close by in comparison to the Bohr radius, or far away". We adapt this premise to the magnetic field case by proposing that at high enough $B$ "close by" means within an area $2 \pi l_{\mathrm{b}}^{2}$, where $l_{\mathrm{b}}$ is the magnetic length $(=\sqrt{\hbar / e B})$, and "far away" means outside that area.

The area $2 \pi l_{\mathrm{b}}^{2}$ is the inverse of the Landau level degeneracy. Since $\nu=$ $n_{\mathrm{e}} h / e B$, the probability that one electron is present in an area $2 \pi l_{\mathrm{b}}{ }^{2}=h / e B$ is equal to $\nu$ up to $\nu=1$ and remains fixed at 1 thereafter. Furthermore, the probability of having two electrons in $2 \pi l_{\mathrm{b}}^{2}$ is 0 up to $\nu=1$, it is $\nu-1$ in the range $1<\nu<2$, and it is fixed at 1 for $\nu \geq 2$. We can formulate the following set of "rules" that the five absorption processes appear to obey, at least approximately, up to $\nu=2$ :

Rule (i): There is a quite accurate sum rule for the total oscillator strength of the five processes $X, X \& C R, T, X \& 2 C R, T \& C R$ in a given polarization; if one process gains intensity, other processes lose intensity. 


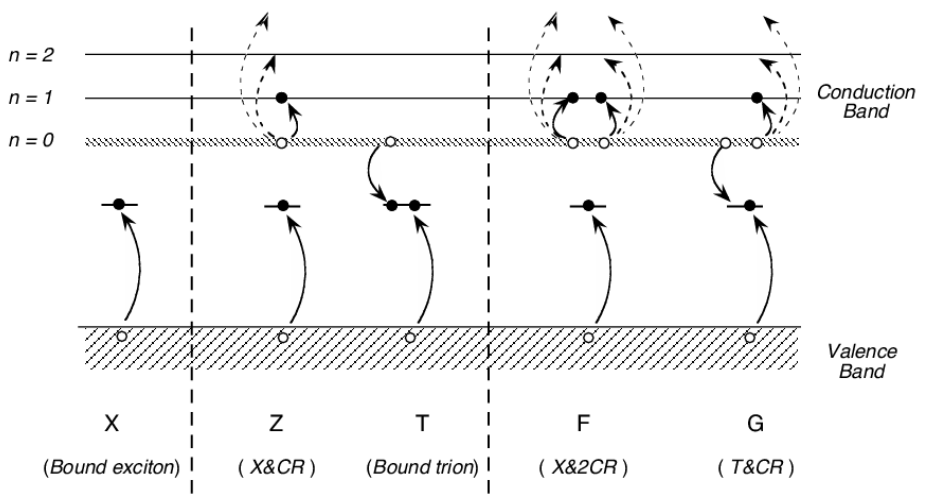

Fig. 5. Exciton, trion, and quatron magneto-absorption processes are shown for filling factor $\nu \leq 2$ (to simplify the drawing, spin states are not distinguished). One or two electrons can be bound by the valence band hole into levels below the conduction band. Shading of lowest Landau level $n=0$ indicates its partial or complete filling by electrons; the trion and quatron processes create, respectively, one and two holes in this level. The five processes share the initial excitonic oscillator strength. In our model for $\nu>2$ (Sec. 3.4), oscillator strength is transferred to the higher cyclotron scatterings (shown by dotted arrows here) as the $n=1,2, \ldots$ Landau levels fill with electrons.

Rule (ii): The combined probability of the two trion processes is $\approx 100 \%$ if there is one electron within an interaction area $2 \pi l_{\mathrm{b}}^{2}$ centred on the exciton at the instant of photon absorption.

Rule (iii): The combined probability of the quatron processes is $\approx 100 \%$ if two electrons are present in an interaction area $2 \pi l_{\mathrm{b}}^{2}$ centred on the exciton at the instant of photon absorption.

Note that two electrons in an area $2 \pi l_{\mathrm{b}}^{2}$ will necessarily have opposite spins, by exclusion. These rules describe our observations rather well for sufficiently high magnetic field $(B \gtrsim 3 \mathrm{~T})$, at low temperature, for example:

At $\nu \geq 1$, there is always an electron within $l_{\mathrm{b}}$ and by Rule (ii), the exciton resonance $X$ disappears (Fig. 2), or almost (Fig. 4). We emphasize that photons still create excitons, but excitons that are scattered or converted into trions (processes illustrated in middle panel of Fig. 5).

In $\sigma^{-}$, the trion resonance $T$ gains intensity up to $\nu=1$ but then $T \& C R$ can start up and takes intensity from $T$ by Rule (i), so the observation of Lovisa et al. [19] that $T$ peaks in intensity at $\nu \approx 1$ is explained without invoking phasespace filling. From $\nu>1$, trions can also be created in the alternate polarization $\sigma^{+}$but, since a second electron is already present within $l_{\mathrm{b}}$, any trion thus formed is always scattered (Rule (iii)). So it is $T \& C R$ that grows in the range $1<\nu<2$, along with $X \& 2 C R$, all these processes sharing intensity as per Rule $(i)$.

At $\nu \geq 2$, there are always two electrons - of opposite spin - within $l_{\mathrm{b}}$ so now all trions created in either polarization are scattered (right hand 
panel in Fig. 5). This explains the extinction/emergence of the $T$ resonance at $\nu=2$ (Fig. 2a, Fig. 4a), again without invoking phase-space filling. Once more we emphasize: At $\nu>2$, photons still create trions, but they are scattered trions.

The presence of an electron (or two electrons) within an interaction area $2 \pi l_{\mathrm{b}}^{2}$ could perhaps be more precisely defined, in the symmetric gauge, as the probability of occupation of the zero angular momentum orbital $m=0$ centred on the exciton. It appears significant that this $2 \mathrm{D}$ Gaussian function of radius $l_{\mathrm{b}}$ is the only one of the $e B / h$ orbitals of Landau index $n=0$ without a node at the origin. The radius $l_{b}$ is, for example, $14.8 \mathrm{~nm}$ at $B=3 \mathrm{~T}$, apparently small enough that an electron in this orbital interacts very efficiently with an exciton of radius $a_{\mathrm{B}}=5 \mathrm{~nm}$.

\subsection{Renormalisation effects}

At low $T$ in a low disorder sample, electron scattering processes at $\nu \leq 2$ must leave a conduction hole in the lowest Landau level, as illustrated in Fig. 5. We propose that this explains why $T \& C R$ crosses the extrapolated line of the $T$ resonance at $B \neq 0$ in Fig. 3, that the interaction of the conduction-electron conduction-hole pair with the trion renormalises the energy of the quatron excitation downwards, so that the extrapolated energy positions of $T \& C R$ and $T$ do not meet at $B=0$.

Likewise, the interaction between a cyclotron excitation and an exciton would explain why $X \& C R$ and $X$ cross at progressively increasing $B$ with increasing $n_{\mathrm{e}}$, as measured but not explained in Ref. [9].

\subsection{Filling $\nu>2$}

We discuss briefly the pattern of peaks $G_{i}$ seen at the lowest fields $(\nu>2)$ in Fig. 2a and b, emerging from the $\omega_{1}$ peak. Peaks labelled ... $G_{3}, G_{2}, G_{1}$ each appear at successively higher fields corresponding to integer values of $\nu$, become strong over a certain range of $\nu$, then fade away. Inversely, varying $n_{\mathrm{e}}$ at fixed low values of $B$ (1-2 T, data not shown here), we see peaks $G_{1}, G_{2}, G_{3}, \ldots$ appearing/fading at successively higher $n_{\mathrm{e}}$.

Previously (e.g. Ref. [20]), we have thought of absorption peaks $G_{i}$ (including $G_{1}$, re-interpreted above as $T \& C R$ ) as "free-carrier transitions $L_{i i}$ ", from valence band Landau levels $i$ to conduction band Landau levels $i$. But the slopes of the graphs of their energy positions against $B$ fit just as well with processes $T \& C R_{i}$, that is to trion creation with electron scattering $i \times \hbar \omega_{\text {ce }}$ from the lowest Landau level $n=0$ to Landau level $n=i$, if trion recoil is included. And the intensities fit with an additional "rule":

Rule (iv): In $T \& C R$ scattering, electrons are excited from the $n=0$ Landau level only, and principally to the lowest or the next lowest unfilled Landau level.

Referring to Fig. 5 (right panel), scattering process $T \& C R_{1}$ will evidently be blocked progressively as the second Landau level $(n=1)$ fills, that is from $\nu \geq 2$. 
From Rule (iv) and the oscillator strength sharing Rule (i), process $T \& C R_{2}$ will then gain intensity in compensation. From $\nu=4$, as the third Landau level $(n=2)$ fills, the process $T \& C R_{2}$ is blocked and its intensity is transferred progressively to $T \& C R_{3}$. And so on. More precisely, with spin splitting of the Landau levels, peaks $T \& C R_{i}$ are extinguished at even and odd integer $\nu$ in $\sigma^{-}$and $\sigma^{+}$, respectively.

\section{Conclusion}

We are led to the conclusion that absorption spectra seen at "low" $n_{\mathrm{e}}$ (low but not very low compared to $1 / \pi a_{\mathrm{B}}^{2}$ ) should be interpreted within a model of a strong exciton hardly affected by phase-space filling and screening. The excitonic transitions retain their oscillator strength almost entirely, but they are shifted slightly in energy to re-appear as the trion resonance or as scattering bands.

In a magnetic field, the quantisation of the electronic momentum and spin helps to resolve five distinct processes: the exciton, two trion, and two quatron processes. Following up on our recent study [9] of oscillator strength sharing between $X, T$, and $X \& C R$ at $\nu<1$, we have now confirmed intensity sharing with the quatron processes $T \& C R$ and $X \& 2 C R$ from $\nu=1$ to $\nu=2$.

As concerns the whole series of peaks $G_{i}$ seen at a higher filling $\nu>2$, we are led (Sec. 3.4) to abandon our early attribution of these peaks to "band to band" transitions. The $T \& C R$ process of Kocheresnko et al. now provides a much more satisfying model at low $n_{\mathrm{e}}$, with the electron scattering going from the $n=0$ Landau level to the lowest empty Landau levels. And if we extrapolate this interpretation to $\nu=\infty$, the broadened zero-field peak $\omega_{1}$ becomes trion creation plus scattering of a single electron from the conduction band edge to the Fermi level.

That would be very different indeed from any interpretation of $\omega_{1}$ in terms of multiple small scatterings at the Fermi level (the Fermi edge singularity). The $\omega_{2}$ absorption peak would then become exciton creation plus scattering of two electrons from the conduction band edge to the Fermi level, and the $\omega_{2}-\omega_{1}$ splitting would contain the difference between the two electron and the one electron scatterings, of the order of $E_{\mathrm{F}}$, plus the recoil term difference.

Next (difficult) tests for the strong exciton model will be explaining the absorption spectra of $p$-type QWs (e.g. Refs. [21, 22]) and, crucially, reconciling this model with QW emission properties, such as the Moss-Burstein shift.

\section{References}

[1] P. Kossacki, J. Phys., Condens. Matter 15, R471 (2003).

[2] D.R. Yakovlev, V.P. Kochereshko, R.A. Suris, H. Schenk, W. Ossau, A. Waag, G. Landwehr, P.C.M. Christianen, J.C. Maan, Phys. Rev. Lett. 79, 3974 (1997).

[3] B. Stébé, E. Feddi, A. Ainane, F. Dujardin, Phys. Rev. B 58, 9926 (1998). 
[4] F.X. Bronold, Phys. Rev. B 61, 12620 (2000).

[5] R.A. Suris, V.P. Kochereshko, G.V. Astakhov, D.R. Yakovlev, W. Ossau, J. Nürnberger, W. Faschinger, G. Landwehr, T. Wojtowicz, G. Karczewski, J. Kossut, Phys. Status Solidi B 227, 343 (2001).

[6] A. Esser, R. Zimmermann, E. Runge, Phys. Status Solidi B 227, 317 (2001).

[7] S. Schmitt-Rink, D.S. Chemla, D.A.B. Miller, Adv. Phys. 38, 89 (1989).

[8] P. Hawrylak, Phys. Rev. B 44, 3821 (1991).

[9] R.T. Cox, R.B. Miller, K. Saminadayar, T. Baron, Phys. Rev. B 69, 235303 (2004).

[10] A. Esser, M.A. Dupertuis, E. Runge, R. Zimmermann, in: IOP Conf. Series 171, Physics of Semiconductors 2002, Proc. 26th Intern. Conf., Edinburgh 2002, Eds. A.R. Long, J.H. Davies, IOP Publishing, Bristol 2003, p. R 4.2.

[11] V.P. Kochereshko, M. Kutrowski, D.A. Andronikov, T. Wojtowicz, G. Karczewski, J. Kossut, Phys. Status Solidi C 0, 1463 (2003); V.P. Kochereshko, D.R. Yakovlev, G.V. Astakhov, R.A. Suris, J. Nurnberger, W. Faschinger, W. Ossau, G. Landwehr, T. Wojtowicz, G. Karczewski, J. Kossut, in: Optical Properties of Semiconductor Nanostructures, NATO Science Series 3, Vol. 81, Eds. M.L. Sadowski, M. Potemski, M. Grynberg, Kluwer, Dordrecht 2000, p. 299.

[12] R. Suris, in: Optical Properties of 2D Systems with Interacting Electrons, NATO Science Series II, Vol. 119, Eds. W.J. Ossau, R. Suris, Kluwer, Dordrecht 2003, p. 111.

[13] V. Huard, R.T. Cox, K. Saminadayar, A. Arnoult, S. Tatarenko, Phys. Rev. Lett. 84, 187 (2000).

[14] G. Yusa, H. Shtrikman, I. Bar-Joseph, Phys. Rev. B 62, 15390 (2000).

[15] R. Kaur, A.J. Shields, J.L. Osborne, M.Y. Simmons, D.A. Ritchie, M. Pepper, Phys. Status Solidi A 175, 465 (2000).

[16] J.A. Brum, P. Hawrylak, Comments Condens. Matter Phys. 18, 135 (1997).

[17] K. Kheng, R.T. Cox, Y. Merle d'Aubigné, Franck Bassani, K. Saminadayar, S. Tatarenko, Phys. Rev. Lett. 71, 1752 (1993).

[18] A. Esser, Ph. D. Thesis, Humboldt University (Berlin), Logos Verlag, Berlin 2002.

[19] S. Lovisa, R.T. Cox, N. Magnea, K. Saminadayar, Phys. Rev. B 56, 12787 (1997).

[20] R.T. Cox, K. Kheng, K. Saminadayar, T. Baron, S. Tatarenko, in: High Magnetic Fields in the Physics of Semiconductors, Ed. D. Heiman, World Scientific, Singapore 1995.

[21] P. Kossacki, J. Cibert, D. Ferrand, Y. Merle d'Aubigné, A. Arnoult, A. Wasiela, S. Tatarenko, J.A. Gaj, Phys. Rev. B 60, 16018 (1999).

[22] P. Płochocka, P. Kossacki, W. Maślana, J. Cibert, S. Tatarenko, C. Radzewicz, J.A. Gaj, Phys. Rev. Lett. 92, 177402 (2004). 